\title{
Scattering Theory for $N$-Particle Systems with Stark Effect: Asymptotic Completeness
}

By

Hideo TAMURA*

\section{$\S 1$. Introduction}

The present paper is a continuation to the work [15] where we have proved the non-existence of bound states and the principle of limiting absorption for $N$-particle Stark Hamiltonians. We here study the problem of asymptotic completeness of wave operators. The asymptotic completeness for $\mathrm{N}$ particle quantum systems without uniform electric fields was first proved by Sigal-Soffer [12] and after that remarkable work, alternative proofs have also been given by several authors $[2,7,13,17]$. We use the local commutator method initiated by Mourre [9] to prove the asymptotic completeness for $N$ particle systems with uniform electric fields. The proof is, in principle, based on the same idea as developed in the above works $[2,7,12,13,17]$ for the case without electric fields. We analyse the propagation properties in the configuration space for solutions to the Schrödinger equation. But the phase space analysis is not required, because charged particles are scattered along only one direction (direction of a given uniform electric field).

We shall formulate the problem precisely, fixing several basic notations employed in many-particle scattering theory. We consider a system of $N$ particles moving in a constant electric field $\mathcal{E} \in \boldsymbol{R}^{3}, \mathcal{E} \pm 0$. We denote by $m_{\jmath}, e_{1}$ and $r_{\jmath} \in \boldsymbol{R}^{3}, 1 \leqq j \leqq N$, the mass, charge and position vector of the $j$-th particle, respectively. Then, for such a system, the total energy Hamiltonian takes the following form:

$$
-\sum_{1 \leqq j \leqq N}\left(\Delta / 2 m_{j}+e_{j}\left\langle\mathcal{E}, r_{\jmath}\right\rangle\right)+V,
$$

where the notation $\langle\cdot, \cdot\rangle$ denotes the usual scalar product in the Euclidean space and the potential $V$ is given as the sum of pair potentials

$$
V=\sum_{1 \leqq j<k \leqq N} V_{\jmath k}\left(r_{j}-r_{k}\right) \text {. }
$$

Communicated by T. Kawai, January 7, 1993. Revised February 8, 1993.

1991 Mathematics Subject Classification: 35P25.

* Department of Mathematics, Ibaraki University, Mito, Ibaraki, 310, Japan. 
For notational brevity, we fix the values of masses throughout as

$$
m_{j}=1, \quad 1 \leqq j \leqq N
$$

but we regard the values of charges as real parameters. As usual, the above operator is considered in the center-of-mass frame. We define the configuration space $X$ as

$$
X=\left\{r=\left(r_{1}, \cdots, r_{N}\right) \in \boldsymbol{R}^{3 \wedge N}: \sum_{1 \leqq j \leqq N} r_{j}=0\right\}
$$

and the vector $E$ as

$$
E=\text { projection onto } X \text { of }\left(e_{1} \mathcal{E}, \cdots, e_{N} \mathcal{E}\right) .
$$

We write $x$ for a generic point in $X$. Then, for the system with identical masses $m_{\jmath}=1$, the energy Hamiltonian $H$ (Schrödinger operator) takes the following form in the center-of-mass frame and acts on the space $L^{2}(X)$ :

$$
H=-\Delta / 2-\langle E, x\rangle+V \quad \text { on } L^{2}(X) .
$$

We assume that $|E| \neq 0$, which means that the charges $e_{j}$ do not take an identical value for all $j$. All the pair potentials $V_{\jmath k}$ are assumed to fulfill the following assumption:

$$
\begin{aligned}
& V_{j_{k}}(y) \in C^{2}\left(\boldsymbol{R}^{3}\right) \text { is a } C^{2} \text {-smooth real function with decay properties } \\
& \left|V_{j k}(y)\right|+\left|\nabla V_{j k}(y)\right|=O\left(|y|^{-o}\right), \quad\left|\nabla \Gamma V_{j_{k}}(y)\right|=O(1) \text { for some } \rho>1 / 2
\end{aligned}
$$

Throughout the entire discussion, the constant $\rho$ is used with the meaning ascribed above. This assumption on pair potentials enables $H$ formally defined above to admit a unique self-adjoint realization in $L^{2}(X)$ with its natural domain. We use the same notation $H$ to denote this self-adjoint realization. In the previous work [15], we have proved the non-existence of bound states and the principle of limiting absorption for the operator $H$ under assumption (V).

The problem of asymptotic completeness is to determine completely the asymptotic states as time $t \rightarrow \pm \infty$ of solutions $\phi(t)=\exp (-i t H) \psi$ to the Schrödinger equation for all initial states $\phi \in L^{2}(X)$. The asymptotic behavior of solutions depends on the values of charges $e_{\jmath}$. Charged particles go to infinity along the direction of electric field in classical mechanics. For example, the $j$-th particle behaves like $e_{j} \mathcal{E} t^{2} / 2$ as $t \rightarrow \pm \infty$ in $\boldsymbol{R}^{3}$. We now define the direction $\omega$ at $\omega=E /|E|$. Then the $N$ particles under consideration go to infinity along direction $\omega$ in the configuration space $X$. If all the charges $e_{j}$ take different values, then the pair potentials $V_{\jmath_{k}}$ all decay along direction $\omega$ in $X ; V_{j_{k}}\left(r_{\jmath}-\right.$ $\left.r_{k}\right) \rightarrow 0$ as $|r| \omega \rightarrow \infty$. Hence the solution $\phi(t)$ can be expected to behave like the free motion $\exp \left(i t H_{0}\right) \psi_{0}^{ \pm}$with some $\psi_{0}^{ \pm} \in L^{2}(X)$ as $t \rightarrow \infty$, where $H_{0}$ denotes the free Hamiltonian

$$
H_{0}=-\Delta / 2-\langle E, x\rangle \text {. }
$$


On the other hand, if, for example, two charges $e_{j}$ and $e_{k}$ take the same value for some pair $(j, k), 1 \leqq j<k \leqq N$, then the $j$-th and $k$-th particles may form a bound state at some energy and go to infinity as $t \rightarrow \infty$, so that the solution $\psi(t)$ has scattering channels associated with such bound states as asymptotic states. Thus the asymptotic behavior of solutions is different according to the values of charges.

We shall discuss the matter more precisely. To do this, we require several basic notations in many-particle scattering theory.

First we denote by letter $a$ or $b$ a partition $a=\left\{C_{1}, \cdots, C_{m}\right\}$ of the total set $\{1, \cdots, N\}$ into non-empty disjoint subsets (clusters) $C_{j}$. Such a partition is called a cluster decomposition. We also denote by $\#(a)$ the number of clusters in $a$. The one-cluster decomposition $a=\{1,2, \cdots, N\}, \#(a)=1$, is not used throughout the whole exposition. We further write $\alpha$ for pair $(j, k)$ with $1 \leqq j<k \leqq V$ and $V_{\alpha}$ for pair potential $V_{j k}$. The relations $\alpha \subset a$ and $\alpha \not \subset a$ mean, respectively, that $j$ and $k$ are in the same cluster in $a$ and that they are in different clusters in $a$. The pair $\alpha=(j, k)$ is sometimes identified with the $N-1$ cluster decomposition $\{(j, k),(1), \cdots,(\hat{j}), \cdots,(\hat{k}), \cdots,(N)\}$. If $b$ is obtained as a refinement of $a$, then we use the same notation $\subset$ to denote this relation as $b \subset a$. We note that $a \subset a$ is regarded as a refinement of $a$ itself.

We define the two subspaces $X^{a}$ and $X_{a}$ of $X$ as

$$
\begin{aligned}
& X^{a}=\left\{r \in X: \sum_{j \in C} r_{j}=0 \text { for all clusters } C \text { in } a\right\}, \\
& X_{a}=\left\{r \in X: r^{n}=r_{j}-r_{k}=0 \text { for all pairs } \alpha \subset a\right\} .
\end{aligned}
$$

These spaces are mutually orthogonal and span the total space $X=X^{a} \oplus X_{a}$, so that $L^{2}(X)$ is decomposed as the tensor product $L^{2}(X)=L^{2}\left(X^{a}\right) \otimes L^{2}\left(X_{a}\right)$. We also denote by $\pi^{a}: X \rightarrow X^{a}$ and $\pi_{a}: X \rightarrow X_{a}$ the orthogonal projections onto $X^{a}$ and $X_{a}$, respectively, and write $x^{a}=\pi^{a} x$ and $x_{a}=\pi_{a} x$ for a generic point $x \in X$.

The cluster Hamiltonian $H_{a}$ is defined by

$$
H_{a}=-\Delta / 2-\langle E, x\rangle+V^{a} \quad \text { on } \quad L^{2}(X)
$$

as an operator acting on $L^{2}(X)$, where

$$
V^{a}(r)=\sum_{\alpha \subset a} V_{\alpha}\left(r^{\alpha}\right), \quad r^{\alpha}=r_{j}-r_{k} .
$$

Let $E^{a}=\pi^{a} E$ and $E_{a}=\pi_{a} E$. Then the operator $H_{a}$ is decomposed into

$$
H_{a}=H^{a} \otimes I d+I d \otimes T_{a} \quad \text { on } \quad L^{2}\left(X^{a}\right) \otimes L^{2}\left(X_{a}\right) .
$$

Here $H^{a}$ is the subsystem Hamiltonian defined by

$$
H^{a}=-\Delta / 2-\left\langle E^{a}, x^{a}\right\rangle+V^{a} \quad \text { on } \quad L^{2}\left(X^{a}\right)
$$

and $T_{a}$ is the free Hamiltonian defined by

$$
T_{a}=-\Delta / 2-\left\langle E_{a}, x_{a}\right\rangle \text { on } L^{2}\left(X_{a}\right) .
$$


Finally we define the set $\Gamma$ as

$$
\Gamma=\left\{a: E^{a}=0,2 \leqq \#(a) \leqq N-1\right\} .
$$

If all the charges $e$, take different values, then $\Gamma=\varnothing$ becomes empty and the converse is also true.

We now return to the problem on the asymptotic behavior of solution $\phi(t)$. If $a \in \Gamma$, then the subsystem operator $H^{a}=-\Delta / 2+V^{a}$ has no electric field, so that it may have bound states in $L^{2}\left(X^{a}\right)$. We denote by $P^{a}: L^{2}\left(X^{a}\right) \rightarrow L^{2}\left(X^{a}\right)$ the eigenprojection associated with $H^{a}$. What we want to prove is that for any initial state $\phi \in L^{2}(X)$, there exist $\phi_{0}^{ \pm}$and $\phi_{a}^{ \pm}, a \in \Gamma$, such that the solution $\phi(t)=\exp (-i t H) \psi$ behaves like

$$
\phi(t)=\exp \left(-i t H_{0}\right) \phi_{0}^{ \pm}+\sum_{a \in \Gamma} \exp \left(-i t H_{a}\right)\left(P^{a} \otimes I d\right) \psi_{a}^{x}+o(1), \quad t \rightarrow \pm \infty,
$$

where the notation $o(1)$ stands for terms converging to zero in $L^{2}(X)$. The first term on the right side denotes the free channel and the second one denotes the scattering channel associated with bound states. If, in particular, $\Gamma=\varnothing$, then the solution behaves like the free motion.

We define the wave operators $W_{a}^{ \pm}: L^{2}(X) \rightarrow L^{2}(X)$ by

$$
W_{a}^{ \pm}=s-\lim _{t \rightarrow \pm \infty} \exp (i t H) \exp \left(-i t H_{a}\right)\left(P^{a} \otimes I d\right)
$$

for $a \in \Gamma$. We further write $W_{0}^{ \pm}=W_{a}^{ \pm}, \#(a)=N$, for the wave operators with $N$-cluster decomposition $\#(a)=N$, which is defined by the relation above with zero eigenprojection $P^{a}=0$. If the wave operators exist, it can be easily verified that the ranges of wave operators are all closed and mutually orthogonal

$$
\text { Range } W_{a}^{ \pm} \perp \text { Range } W_{b}^{ \pm}, \quad a \pm b .
$$

The problem of asymptotic completeness is now formulated as

Problem (asymptotic completeness).

$$
L^{2}(X)=\text { Range } W_{0}^{ \pm} \oplus \sum_{a \in \Gamma} \text { Range } W_{a}^{ \pm} .
$$

In order to answer this question, we add the following assumption to (V). (VS) $V_{\alpha}(y)=O\left(|y|^{-d}\right), d>1$, is short-range for $\alpha \subset a$ with $a \in \Gamma$.

We are now in a position to state the main theorem obtained in this work.

Theorem 1.1. Let the notations be as above. Assume that (V) and (VS) are fulfilled. Then the wave operators $W_{0}^{ \pm}$and $W_{a}^{ \pm}, a \in \Gamma$, exist and are asymptotically complete.

The proof of the theorem uses the asymptotic completeness for $H^{a}$ with $a \in \Gamma$. For such a cluster decomposition $a$, the subsystem operator $H^{a}$ does 
not have a uniform electric field and hence this result has been obtained by [12] under assumption (VS). For this reason, (VS) is assumed.

We conclude the section by making a brief comment on the results related to the main theorem. The asymptotic completeness for Stark Hamiltonians has been already studied by many authors $[1,3,4,5,10,16,18]$ for one or twoparticle systems, including the case of scattering by long-range potentials. On the other hand, there are only a few works dealing with the scattering problem for many-particle systems. In his work [8], Korotyaev first proved the completeness of wave operators for three-particle systems. The proof is based on the Faddeev equation method. The restrictive smoothness condition on pair potentials as in $(\mathrm{V})$ is not assumed there. But some additional assumption is imposed. The assumption is stated in terms of the notations here as follows: $V_{\alpha}$ satisfies (VS) with $d>2$ for $\alpha \subset a, a \in \Gamma$, $a$ being a two-cluster decomposition, and the two-particle subsystem operator $H^{a}=-\Delta / 2+V_{\alpha}$ acting on $L^{2}\left(\boldsymbol{R}^{3}\right)$ does not have a resonance state at zero energy. After Korotyaev's work [8], the author [14] has given an alternative proof based on the local commutator method without assuming the resonance condition as above.

\section{$\S 2$. Existence of Wave Operators}

In this section, we prove the existence of wave operators $W_{0}^{ \pm}$and $W_{a}^{ \pm}, a \in$ $\Gamma$, defined by (1.4). The proof uses only the decay property of pair potentials $V_{\alpha}$ but does not require the other decay properties of derivatives.

Proposition 2.1. Assume that (V) and (VS) are satisfied. Then the wave operators $W_{0}^{ \pm}$and $W_{a}^{ \pm}, a \in \Gamma$, exist and their ranges are all closed and mutually orthogonal.

Proof. We prove only the existence of wave operator $W_{a}^{+}$with $a \in \Gamma$. A similar argument applies to the other operators and the properties (closedness and orthogonality) of ranges can be also easily verified.

We denote by $\|\cdot\|_{0}$ the $L^{2}$ norm in $L^{2}(X)$. To prove the proposition, it suffices by the Cook-Kuroda method to show that

$$
J(t)=\left\|V_{\alpha}\left(r^{\alpha}\right) \exp \left(-i t H_{a}\right) \psi\right\|_{0} \in L^{1}((1, \infty)), \quad \alpha \not \subset a,
$$

as a function of $t>1$ for $\psi$ in a fundamental set of $L^{2}(X)$. We define the set $\mathscr{D}$ as

$$
\mathscr{D}=\left\{f\left(H^{a}\right) \theta^{a} \otimes \theta_{a}: \theta^{a} \in \mathcal{S}\left(X^{a}\right), \hat{\theta}_{a} \in C_{0}^{\infty}\left(X_{a}^{\prime}\right), f \in C_{0}^{\infty}(\boldsymbol{R})\right\},
$$

where $\mathcal{S}\left(X^{a}\right)$ is the Schwartz space over $X^{a}, \hat{\theta}_{a}$ is the Fourier transform of $\theta_{a} \in \mathcal{S}\left(X_{a}\right)$ and $X_{a}^{\prime}$ denotes the space dual to $X_{a}$. Then it is easily seen that $\mathscr{D}$ becomes a fundamental set of $L^{2}(X)$ and also we have 


$$
\exp \left(-i t H_{a}\right) \psi=\exp \left(-i t H^{a}\right) f\left(H^{a}\right) \theta^{a} \otimes \exp \left(-i t T_{a}\right) \theta_{a}
$$

for $\phi \in \mathscr{D}$.

For brevity, we now fix $a \in \Gamma$ as $a=\left\{C_{1}, C_{2}, \cdots, C_{l}\right\}, 2 \leqq l \leqq N-1$, and assume that $j \in C_{1}$ and $k \in C_{2}$ for pair $\alpha=(j, k)$. We denote by $\zeta_{0} \in \boldsymbol{R}^{3}$ the intercluster coordinates with normalization between clusters $C_{1}$ and $C_{2}$ and write $\zeta=\left(\zeta_{0}, \zeta^{\prime}\right)=\left(\zeta_{0}, \zeta_{1}, \cdots, \zeta_{l-2}\right)$ for the normalized Jacobi coordinate system over $X_{a}$. Let $\#(C)$ be the number of particles in cluster $C$. We also choose $\hat{\xi}=\left(\xi_{1}, \cdots, \hat{\xi}_{m}\right), m=\#\left(C_{1}\right)+\#\left(C_{2}\right)-2$, as coordinates within clusters $C_{1}$ and $C_{2}$. Then the relative coordinates $r^{a}$ are represented as a linear combination of the form

$$
r^{n}=c_{0} \zeta_{0}+\sum_{1 \leqq i \leqq m} d_{i} \tilde{\xi}_{2}, \quad c_{0} \neq 0,
$$

and $E_{a}$ has the representation $E_{a}=\left(F_{0}, F^{\prime}\right)=\left(F_{0}, F_{1}, \cdots, F_{l-2}\right)$ in terms of the coordinate system $\zeta$ over $X_{a}$.

We define $T_{0}$ by $T_{0}=-\Delta / 2$ as an operator acting on $L^{2}\left(X_{a}\right)$ and set

$$
\theta_{a t}\left(x_{a}\right)=\exp \left(-i t T_{0}\right) \theta_{a}\left(x_{a}\right) .
$$

We now use Proposition 19.1 of [10] which gives the relation between the propagators $\exp \left(-i t T_{a}\right)$ and $\exp \left(-i t T_{0}\right)$. According to this relation, we have

$$
\left|\theta_{a t}\left(x_{a}-E_{a} t^{2} / 2\right)\right|=\left|\exp \left(-i t T_{a}\right) \theta_{a}\left(x_{a}\right)\right| \text {. }
$$

Hence we make a change of variables to obtain that

$$
J(t)^{2}=\int\left|V_{\alpha}\left(r^{\alpha}+c_{0} F_{0} t^{2} / 2\right)\right|^{2}\left|\theta_{t}^{a}\left(x^{a}\right)\right|^{2}\left|\theta_{a t}\left(x_{a}\right)\right|^{2} d x,
$$

where $r^{a}$ is represented as in (2.2) and

$$
\theta_{t}^{a}\left(x^{a}\right)=\exp \left(-i t H^{a}\right) f\left(H^{a}\right) \theta^{a}\left(x^{a}\right), \quad f \in C_{0}^{\infty}(\boldsymbol{R}) .
$$

If $\alpha \subset b$ for some $b \in \Gamma$, then $F_{0}=0$ and also $V_{a}$ is a short-range potential by assumption (VS). In this case, we can prove (2.1) in exactly the same way as in the proof of Theorem XI. 34 in [11]. Thus we assume that $a \not \subset b$ for any $b \in \Gamma$, so that $F_{0} \neq 0$ never vanishes.

Lemma 2.2. Let $\theta_{t}^{a}$ be as above. Let $\varepsilon>0$ be fixed arbitrarily but small enough. Then

$$
\int_{\left|x^{a}\right|>\varepsilon|t|^{2}}\left|\theta_{t}^{a}\left(x^{a}\right)\right|^{2} d x^{a}=O\left(t^{-2 \nu}\right), \quad t \longrightarrow \infty,
$$

for some $\nu>1$.

We complete the proof of the proposition, accepting the lemma above as proved. Since $\hat{\theta}_{a} \in C_{0}^{\infty}\left(X_{a}^{\prime}\right)$ is supported in $\left\{p_{a} \in X_{a}^{\prime}:\left|p_{a}\right|<R\right\}$ for some $R>0$, it follows that 


$$
\left|\theta_{a t}\left(x_{a}\right)\right| \leqq C_{L}\left(1+t+\left|x_{a}\right|\right)^{-L}, \quad L \gg 1,
$$

for $\left|x_{a}\right|>2 R t$. Thus we decompose $J(t)^{2}$ into the three integrals;

$$
J(t)^{2}=\left\{\int_{\left|x_{a}\right|>2 R t}+\int_{\left|x a_{1>\varepsilon|t|}\right|^{2},\left|x_{a}\right|<2 R t}+\int_{\left|x^{a}\right|<\varepsilon|t|^{2},\left|x_{a}\right|<2 R t}\right\} \cdots d x .
$$

By (2.3) and Lemma 2.2, the first two terms on the right side are of order $O\left(t^{-2 \nu}\right)$ as $t \rightarrow \infty$ for some $\nu>1$. If $x=\left(x^{a}, x_{a}\right)$ is in the integral domain of the third term, then it follows that

$$
\left|r^{\alpha}+c_{0} F_{0} t^{2} / 2\right|>d t^{2}, \quad d>0,
$$

for $t \gg 1$ large enough, so that

$$
\left|V_{\alpha}\left(r^{\alpha}+c_{0} F_{0} t^{2}\right)\right|^{2}=O\left(t^{-4 \rho}\right), \quad t \longrightarrow \infty,
$$

by assumption $(V)$. This proves (2.1) and completes the proof.

Proof of Lemma 2.2. The lemma is easy to prove. We first note that $H^{a}=-\Delta / 2+V^{a}$ does not have a uniform electric field by assumption $a \in \Gamma$. We can easily show that

$$
\left\|\left\langle x^{a}\right\rangle^{2} \exp \left(-i t H^{a}\right) f\left(H^{a}\right)\left\langle x^{a}\right\rangle^{-2}\right\|=O\left(t^{2}\right), \quad t \longrightarrow \infty,
$$

as an operator from $L^{2}\left(X^{a}\right)$ into itself, where $\langle x\rangle=\left(1+|x|^{2}\right)^{1 / 2}$. This proves the lemma with $\nu=2$.

\section{§ 3. Propagation Estimate I}

We again denote by $\|\cdot\|_{\theta}$ the $L^{2}$ norm in $L^{2}(X)$ and write $(\cdot, \cdot)_{0}$ for the scalar product. We say that an operator $B$ acting on $L^{2}(X)$ is locally $H$-smooth, if $B f(H): L^{2}(X) \rightarrow L^{2}(X)$ is bounded for $f \in C_{0}^{\infty}(\boldsymbol{R})$ and if

$$
\int_{-\infty}^{\infty}\|B \exp (-i t H) f(H) \psi\|_{0}^{2} d t \leqq C_{f}\|\phi\|_{0}^{2}
$$

for $\phi \in L^{2}(X)$. In the previous work $[15]$, we have proved the following

Theorem 3.1 (principle of limiting absorption). Assume that $(V)$ is fulfilled. Denote by $L_{2}^{2}(X)=L^{2}\left(X ;\langle x\rangle^{2 \nu} d x\right)$ the weighted $L^{2}$ space over $X$ with weight $\langle x\rangle^{\nu}$. Let $R(\zeta ; H)=(H-\zeta)^{-1}, \operatorname{Im} \zeta \neq 0$, be the resolvent of $H$. Then the resolvents have the boundary values to the real axis

$$
\lim _{\varepsilon \downarrow 0} R(\lambda \pm i \varepsilon ; H)=R(\lambda \pm i 0 ; H), \quad \lambda \in \boldsymbol{R},
$$

in the uniform topology as an operator from $L_{\nu}^{2}(X)$ into $L_{-\nu}^{2}(X)$ for $\nu>1 / 4$, where the convergence is locally uniform in $\lambda$.

From this theorem, we can obtain the following proposition as a consequence 
of the local smoothness theorem due to Kato $([6,11])$.

Proposition 3.2. Assume that $(V)$ is satisfied. Let $z=\langle x, \omega\rangle$. Then the three operators $\langle x\rangle^{-\nu},\langle x\rangle^{-\nu}\langle z\rangle^{-1 / 2} \nabla$ and $\langle x\rangle^{-\nu}\langle z\rangle^{-1} \nabla V$ are all locally H-smooth for any $\nu>1 / 4$.

We here note that the smoothness property of the second and third operators above respectively follows from the $L^{2}$ boundedness of the operators

$$
\langle z\rangle^{-1 / 2} \nabla(H+i)^{-1}, \quad\langle z\rangle^{-1} \nabla V(H+i)^{-1}: L^{2}(X) \longrightarrow L^{2}(X) .
$$

We now define the operator $\gamma$ as

$$
\gamma=\left(D_{0}+D_{0}^{*}\right) / 2, \quad D_{0}=-i\langle x /\langle x\rangle, \nabla\rangle,
$$

and $D_{\perp}$ as

$$
D_{\perp}=-i \nabla-\langle x\rangle^{-1 / 2} \gamma\langle x\rangle^{-1 / 2} x
$$

The aim here is to prove the following

Proposition 3.3. Assume that $(V)$ is satisfied. Let $D_{\perp}$ be defined above. Then the operator $\langle x\rangle^{-3 / 4} D_{\perp}$ is locally $H$-smooth.

Proof. Throughout the proof, we denote by $Q_{\sigma}$ the multiplication operator by $\langle x\rangle^{\sigma}$. We also write $B_{s m}$ for locally $H$-smooth operators and denote by $K_{s m}$ operators represented as $K_{s m}=\sum_{f \imath n i t e} B_{s m}^{*} B_{s m}$.

Define the operator $\Phi$ as $\Phi=Q_{-1 / 4} \gamma Q_{-1 / 4}$ and set

$$
\phi(t)=\exp (-i t H) f(H) \psi, \quad f \in C_{0}^{\infty}(\boldsymbol{R}) .
$$

Then we have

$$
(d / d t)(\Phi \psi(t), \phi(t))_{0}=(i[H, \Phi] \psi(t), \psi(t))_{0} .
$$

Since $\Phi(H+i)^{-1}: L^{2}(X) \rightarrow L^{2}(X)$ is bounded and since

$$
Q_{-3 / 4}\left\langle D_{\perp}, D_{\perp}\right\rangle Q_{-3 / 4}=Q_{-3 / 4}\left(-\Delta-\gamma^{2}\right) Q_{-3 / 4}+K_{s m}
$$

by Proposition 3.2, it suffices to show that

$$
i[H, \Phi] \geqq c Q_{-3 / 4}\left(-\Delta-\gamma^{2}\right) Q_{-3 / 4}+K_{s m}
$$

for some $c>0$ in the form sense over $L^{2}(X)$.

Let $\omega=E /|E|$ be again the direction of $E$. Then $H$ can be rewritten as

$$
H=-\Delta / 2-|E| z+V, \quad z=\langle x, \omega\rangle,
$$

so that we have

$$
i[H, \Phi]=i[-\Delta / 2, \Phi]+i[-|E| z, \Phi]+i[V, \Phi] .
$$


By assumption $(V)$, it follows from Proposition 3.2 that the third operator on the right side takes the form $i[V, \Phi]=K_{s m}$. The second operator is calculated as

$$
i[-|E| z, \Phi]=Q_{-3 / 4}|E| z Q_{-3 / 4}=Q_{-3 / 4}(-\Delta / 2-H+V) Q_{-3 / 4}
$$

and hence

$$
i[-|E| z, \Phi]=Q_{-3 / 4}(-\Delta / 2) Q_{-3 / 4}+K_{s m}
$$

by Proposition 3.2. Finally we calculate the first operator on the right side of (3.4). Since

$$
\begin{aligned}
& i\left[-\Delta / 2, Q_{-1 / 4}\right] \gamma Q_{-1 / 4}=-(1 / 4) Q_{-3 / 4} \gamma^{2} Q_{-3 / 4}+K_{s m}, \\
& Q_{-1 / 4} i[-\Delta / 2, \gamma] Q_{-1 / 4}=Q_{-3 / 1}\left(-\Delta-\gamma^{2}\right) Q_{-3 / 1}+K_{s m}
\end{aligned}
$$

by Proposition 3.2, we obtain that

$$
i[-\Delta / 2, \Phi]=Q_{-3 / 4}\left(-\Delta-3 \gamma^{2} / 2\right) Q_{-3 / 4}+K_{s m} .
$$

This, together with (3.5), gives

$$
i[H . \Phi]=(3 / 2) Q_{-3 / 4}\left(-\Delta-\gamma^{2}\right) Q_{-3 / 4}+K_{s m}
$$

and hence (3.3) follows at once. Thus the proof is complete.

For later use, we here mention one result obtained as an application of Proposition 3.3. Let $q \in C^{\infty}(X)$ be a smooth real function such that $q(x)$ is homogeneous of degree zero for $|x|>1$. We may write $q$ as $q(x)=\chi(x) q_{0}(x /|x|)$ with $q_{0} \in C^{\infty}\left(S_{X}\right), S_{X}$ being the unit sphere in $X$, where $\chi \in C^{\infty}(X)$ vanishes in a small neighborhood of the origin and $\chi=1$ for $|x|>1 / 2$. Then a simple calculation yields that

$$
i[-\Delta / 2, q]=Q_{-1 / 4}\left\{\chi Q_{-3 / 1}\left\langle V_{\theta} q_{0}, D_{\perp}\right\rangle+B_{s m}\right\}
$$

by Proposition 3.2, where $V_{0}$ denotes the gradient over $S_{x}$. Therefore, this relation, together with Proposition 3.3, implies the following

Corollary 3.4. Let $q \in C^{\infty}(X)$ be as above. Then $\left.\langle x\rangle^{1 / 1}[\lrcorner, q\right]$ is locally $H$ smooth.

We end the section by noting that all the results obtained here remain true for cluster Hamiltonians $H_{a}$ under assumption (V).

\section{§4. Propagation Estimate II}

In this section, we establish another type of propagation estimate. We begin by introducing several new notations required to formulate it. We first 
define the two sets $\Lambda, \Lambda^{c}$ of cluster decompositions as

$$
\begin{aligned}
& \Lambda=\left\{a: E_{a}=\pi_{a} E=0,3 \leqq \#(a) \leqq N\right\}, \\
& \Lambda^{c}=\left\{a: E_{a} \neq 0,3 \leqq \#(a) \leqq N\right\} .
\end{aligned}
$$

And we set $\hat{\omega}_{a}=E_{a} /\left|E_{a}\right| \in S_{X}$ for $a \in \Lambda^{c}$. Let $q \in C^{\infty}(X)$ be again a smooth real function such that $q(x)$ is homogeneous of degree zero for $|x|>1$. For such a function, we also define

$$
\text { con supp } q=\left\{\overline{\left.\theta \in S_{X}: q(x)=q(|x| \theta) \neq \dot{\neq} 0,|x|>1\right\}} .\right.
$$

We further define the subset $S_{a}, 2 \leqq \#(a) \leqq N$, of $S_{X}$ as

$$
S_{a}=\left\{\theta=\left(\theta_{1}, \cdots, \theta_{N}\right) \in S_{X}: \theta_{j}=\theta_{k} \text { for } \alpha \subset a, \theta_{j} \neq \theta_{k} \text { for } \alpha \not \subset a\right\} .
$$

By definition, we can easily see that $\left\{S_{a}\right\}$ is a family of disjoint subsets and $S_{X}=\cup_{a} S_{a}$. In particular, the distance $\operatorname{dist}_{X}\left(S_{a}, S_{b}\right)$ over $S_{X}$ between $S_{a}$ and $S_{b}$ is strictly positive for different two-cluster decompositions $a$ and $b$, \# $(a)=$ $\#(b)=2$.

The aim of the present section is to prove the following

Proposition 4.1. Let the notations be as above. Assume that $(V)$ is fulfilled. Let $q \in C^{\infty}(X)$ be a smooth real function such that $q(x)$ is homogeneous of degree zero for $|x|>1$ and that

$$
\begin{array}{ll}
\operatorname{dist}_{X}\left(\operatorname{con} \operatorname{supp} q, S_{a}\right)>d_{0} & \text { for any } a, \#(a)=2, \\
\operatorname{dist}_{X}\left(\operatorname{con} \operatorname{supp} q, \hat{\omega}_{a}\right)>d_{0} & \text { for any } a \in \Lambda^{c}
\end{array}
$$

with some $d_{0}>0$. Then the operator $\langle x\rangle^{-1 / 4} q$ is locally H-smooth.

Proof. Let $\lambda \in \boldsymbol{R}$ be fixed arbitrarily. We take a real-valued function $g \in$ $C_{0}^{\infty}(\boldsymbol{R})$ such that $g$ is supported in a small interval around $\lambda$. To prove the proposition, it suffices to show that the operator $\langle x\rangle^{-1 / 4} q g(H)$ has the $H$ smoothness property. The proof is divided into several steps. Throughout the proof, we use the notations $Q_{\sigma}, B_{s m}$ and $K_{s i n}$ with the same meanings as in the proof of Proposition 3.3.

(1) The first step toward the proof is to construct a partition of unity $\left\{k_{a}\right\}, 2 \leqq \#(a) \leqq N$, over $X$ with the following properties.

(k.1) $k_{a} \in C^{\infty}(X), k_{a} \geqq 0$, is homogeneous of degree zero for $|x|>1$.

(k.2) $\sum_{a} k_{a}(x)^{2}=1$ over $X$.

(k.3) con supp $k_{a} \cap$ con supp $k_{b} \neq \varnothing \Rightarrow a \subset b$ or $b \subset a$.

(k.4) $S_{a} \cap$ con supp $k_{b} \neq \varnothing \Rightarrow a \subset b$ and hence $S_{a} \subset \cup_{a \subset b}$ con supp $k_{b}$.

(k.5) con supp $k_{a} \subset\left\{\theta \in S_{X}:\left|\theta^{a}\right|<\delta\right\}$ for $\delta>0$ small enough. 
Here $\theta^{a}=\pi^{a} \theta$ is the projection onto $X^{a}$ of $\theta$ and the choice of $\delta>0$ depends on the value of $d_{0}$ in (4.1). Such a partition can be easily constructed by use of the simple geometrical properties of the family $\left\{S_{a}\right\}$. By construction, it follows from properties $(k .3)$ and $(k .4)$ that

$$
k_{a}(x) V_{\alpha}\left(r^{a}\right)=O\left(|x|^{-\rho}\right), \quad|x| \longrightarrow \infty,
$$

for $\alpha \not \subset a$. By assumption (4.1), we can also take $\delta$ so small that

$$
\text { con supp } q \cap \text { con supp } k_{a}=\varnothing
$$

for any two-cluster decomposition $a$ and hence we have

$$
q(x)^{2}=\sum_{a} q_{a}(x)^{2}, \quad q_{a}=k_{a} q,
$$

where the sum is taken over $a$ with $3 \leqq \#(a) \leqq N$. By definition, the function $q_{a}$ is homogeneous of degree zero for $|x|>1$.

(2) Let $\Lambda, \Lambda^{c}$ and $g \in C_{0}^{\infty}(\boldsymbol{R})$ be as above. If $a \in \Lambda$, then $\omega=\omega^{a}$ and hence $z=\langle x, \omega\rangle=\left\langle x^{a}, \omega^{a}\right\rangle$ becomes a coordinate over $X^{a}$. In the previous work [15], we have constructed a conjugate operator $A^{a}, a \in \Lambda$, with the following properties. ( $A .1) A^{a}$ is a differential operator acting on $X^{a}$ and takes the form

$$
A^{a}=-i\left\{\left\langle c^{a}\left(x^{a}\right), \nabla^{a}\right\rangle+\left\langle\nabla^{a}, c^{a}\left(x^{a}\right)\right\rangle\right\}+c_{0}^{a}\left(x^{a}\right),
$$

where $\nabla^{a}$ denotes the gradient notation over $X^{a}$ and the coefficients $c^{a}\left(x^{a}\right)=$ $\left\{c_{j}^{a}\left(x^{a}\right)\right\}, 1 \leqq j \leqq 3(N-\#(a))$, and $c_{0}^{a}\left(x^{a}\right)$ are smooth real functions obeying the estimates

$$
\left|\partial^{3} c_{,}^{a}\left(x^{a}\right)\right| \leqq C_{\beta}\langle z\rangle^{-|\beta|}, \quad\left|\partial^{\beta} c_{0}^{a}\left(x^{a}\right)\right| \leqq C_{\beta}\langle z\rangle^{-1-|\beta|} .
$$

(A.2) There exists $d_{a}>0$ such that

$$
g\left(H^{a}+\theta\right) i\left[H^{a}, A^{a}\right] g\left(H^{a}+\theta\right) \geqq d_{a} g\left(H^{a}+\theta\right)^{2}
$$

for $\theta \geqq 0$, where the inequality is understood in the form sense over $L^{2}\left(X^{a}\right)$. We regard $A^{a}$ as an operator over $X$ as well as over $X^{a}$. For $a \in \Lambda$, the operator $T_{a}=-\Delta / 2$ does not have a uniform electric field and also we have $\left[H_{a}, A^{a}\right]=\left[H^{a}, A^{a}\right]$. Thus it follows that

$$
g\left(H_{a}\right) i\left[H_{a}, A^{a}\right] g\left(H_{a}\right) \geqq d_{a} g\left(H_{a}\right)^{2}
$$

for $a \in \Lambda$ in the form sense over $L^{2}(X)$. If $a \in \Lambda^{c}$, then $E_{a}$ does not vanish and hence we can define the operator $\gamma_{a}$ by

for $a \in \Lambda^{c}$.

$$
\gamma_{a}=-i\left\langle\hat{\omega}_{a}, \nabla\right\rangle, \quad \hat{\omega}_{a}=E_{a} /\left|E_{a}\right|,
$$

Let $A^{a}$ and $\gamma_{a}$ be as above. We now define $\Phi$ as $\Phi=\Phi_{1}+\Phi_{2}$, where

$$
\Phi_{1}=\sum_{a \in A^{c}} Q_{-1 / 4} q_{a} \gamma_{a} q_{a} Q_{-1 / 4}, \quad \Phi_{2}=\sum_{a \in A} Q_{-1 / 4} q_{a} \cdot 4^{a} q_{a} Q_{-1 / 4} .
$$


We assert that

$$
g(H) i[H, \Phi] g(H) \geqq d g(H) Q_{-1 / 4} q^{2} Q_{-1 / 4} g(H)+K_{s m}
$$

for some $d>0$. If this assertion can be verified, then the proposition follows at once.

(3) Recall that $H$ is written as

$$
H=-\Delta / 2-|E| z+V, \quad z=\langle x, \omega\rangle .
$$

We again calculate the commutator $i\left[H, \Phi_{1}\right]$ as

$$
i\left[H, \Phi_{1}\right]=i\left[-\Delta / 2, \Phi_{1}\right]+i\left[-|E| z, \Phi_{1}\right]+i\left[V, \Phi_{1}\right] .
$$

Since $\gamma_{a}$ is a differential operator acting on the space $X_{a}$, we have the relation $\left[V_{a}, \gamma_{a}\right]=0$ for $\alpha \subset a$. Hence the third operator on the right side of (4.6) takes the form

$$
i\left[V, \Phi_{1}\right]=K_{s m l}
$$

by (4.2) and Proposition 3.2. A simple calculation gives

$$
i\left[z, \gamma_{a}\right]=-\left|\omega_{a}\right|, \quad \omega_{a}=\pi_{a} \omega,
$$

so that we have

$$
i\left[-|E| z, \Phi_{1}\right]=\sum_{a \in \Lambda^{c}} Q_{-1 / 1} q_{a}\left|E_{a}\right| q_{a} Q_{-1 / 1} .
$$

(4) We evaluate the first operator on the right side of (4.6). Let $\gamma$ be defined by (3.1). Then we have

$$
i\left[-\Delta / 2, Q_{-1 / 1}\right] q_{a} \gamma_{a} q_{a} Q_{-1 / 4}=-(1 / 4) Q_{-3 / 1} q_{a} \gamma \gamma_{a} q_{a} Q_{-3 / 1}+K_{s m}
$$

by Proposition 3.2. We can also write

$$
Q_{-1 / 1} i\left[-\Delta / 2, q_{a}\right] \gamma_{a} q_{a} Q_{-1 / 4}=B_{s m} \gamma_{a} q_{a} Q_{-3 / 4}+K_{s m}
$$

by Corollary 3.4. Since

$$
B_{s m} G+G^{*} B_{s m}^{*} \geqq-\varepsilon G^{*} G+K_{s m}
$$

for any $\varepsilon>0$ small enough and since

$$
-(1 / 4) Q_{-3 \prime^{\prime}}\left(\gamma \gamma_{a}+\gamma_{a} \gamma\right) Q_{-3 / 1} \geqq Q_{-3 / 1}(\Delta / 2) Q_{-3 / 1}+K_{s m},
$$

it follows that

$$
i\left[-\Delta / 2, \Phi_{1}\right] \geqq(1+\varepsilon) \sum_{a \in A^{c}} Q_{-3 / 4} q_{a}(\Delta / 2) q_{a} Q_{-3 / 1}+K_{s m} .
$$

We now use the relation $\Delta / 2=-|E| z-H+V$ to obtain that

$$
i\left[-\Delta / 2, \Phi_{1}\right] \geqq-(1+\varepsilon) \sum_{a \in I c} Q_{-1 / 4} q_{a}\langle E, x /\langle x\rangle\rangle q_{a} Q_{-1 / 1}+K_{s m} .
$$


This, together with (4.7) and (4.8), implies that

$$
i\left[H, \Phi_{1}\right] \geqq \sum_{a \in A^{c}} Q_{-1 / 4} q_{a}\left\{\left|E_{a}\right|-(1+\varepsilon)\langle E, x /\langle x\rangle\rangle\right\} q_{a} Q_{-1 / 4}+K_{s m}
$$

for any $\varepsilon>0$ small enough.

(5) We shall show that the number in brackets on the right side of (4.9) can be made strictly positive for $|x| \gg 1$ with $x \in \operatorname{supp} q_{a}$, if $\delta$ in (k.5) and $\varepsilon$ are chosen small enough. By assumption (4.1), there exists $c>0$ such that $1-\left|\left\langle\hat{\omega}_{a}, \theta\right\rangle\right| \geqq c$ for $\theta \in \mathrm{con} \operatorname{supp} q_{a}, q_{a}=k_{a} q$. We now write $\langle E, x /\langle x\rangle\rangle$ as

$$
\langle E, x /\langle x\rangle\rangle=\left\langle E^{a}, x^{a} /\langle x\rangle\right\rangle+\left\langle E_{a}, x_{a} /\langle x\rangle\right\rangle
$$

and take $\delta$ so small that

$$
|\langle E, x /\langle x\rangle\rangle| \leqq\left|E_{a}\right|\left(\left|\left\langle\hat{\omega}_{a}, \theta\right\rangle\right|+c / 5\right)
$$

for $|x| \gg 1$ with $\theta=x /|x| \in$ con $\operatorname{supp} k_{a}$. Thus we can make the number in question strictly positive by choosing $\varepsilon$ small enough and hence it follows that

$$
g(H) i\left[H, \Phi_{1}\right] g(H) \geqq d \sum_{a \in 1 c} g(H) Q_{-1 / 4} q_{a}^{2} Q_{-1 / 1} g(H)+K_{s m}
$$

for some $d>0$.

(6) The proof is completed in this step. We shall prove a similar form inequality for the commutator $\left[H, \Phi_{2}\right]$. We note that

$$
-(1 / 4) Q_{-3 / 4}\left(\gamma 4^{a}+A^{a} \gamma\right) Q_{-3 / 4} \geqq c Q_{-3 / 4}(\Delta / 2) Q_{-3 / 4}+K_{s m}
$$

for some $c>0$. Hence, by making use of the same argument as in step (4), we obtain that

$$
i\left[H, \Phi_{2}\right] \geqq \sum_{a \in A} Q_{-1 / 4} q_{a}\left\{i\left[H_{a}, A^{a}\right]-c\langle E, x /\langle x\rangle\rangle\right\} q_{a} Q_{-1^{\prime} 4}+K_{s m}
$$

with another $c>0$. By Lemmas 4.4 and 4.5 of [14], the operators

$$
Q_{1 / 2}\left(g(H)-g\left(H_{a}\right)\right) q_{a}, \quad Q_{1 / 2}\left[g\left(H_{a}\right), q_{a} Q_{-1 / 1}\right] Q_{1 / 1}: L^{2}(X) \longrightarrow L^{2}(X)
$$

are bounded and also we can prove that $Q_{1 / 1} g\left(H_{a}\right)\left[H_{a}, A^{a}\right] Q_{-1 / 1}$ is bounded. Thus it follows from (4.4) and Proposition 3.2 that

$$
g(H) i\left[H, \Phi_{2}\right] g(H) \geqq \sum_{a \in A} g(H) Q_{-1 / 1} q_{a}\left\{d_{a}-c\langle E, x /\langle x\rangle\rangle\right\} q_{a} Q_{-1 / 4} g(H)+K_{s m}
$$

for some $d_{a}>0$. Since $E_{a}=0$ for $a \in \Lambda$, we can show, repeating the same argument as used in step (5), that the number in brackets on the right side can be made strictly positive for $|x| \gg 1$ with $x \in \operatorname{supp} q_{a}$. 'This, together with (4.10), proves (4.5) and the proof is now completed.

We conclude the section by noting that the above proposition again remains true for cluster Hamiltonians $H_{a}$. 


\section{$\S 5$. Asymptotic Completeness}

In this section, we complete the proof of the main theorem (Theorem 1.1). Let $f \in C_{0}^{\infty}(\boldsymbol{R})$ and set

$$
\phi(t)=\exp (-i t H) f(H) \psi
$$

for $\phi \in L^{2}(X)$. The $N$-particle system $H$ under consideration is said to be asymptotically clustering, if

$$
\phi(t)=\sum_{a} \exp \left(-i t H_{a}\right) \phi_{a}^{ \pm}+o(1), \quad t \longrightarrow \pm \infty,
$$

for some $\phi_{a}^{ \pm} \in L^{2}(X)$, where the sum is taken over all cluster decompositions $a$ with $2 \leqq \#(a) \leqq N$.

Proposition 5.1. Assume that (V) is fulfilled. Then the $\mathrm{V}$-particle system $H$ is asymptotically clustering in the sense above.

Before going into the proof, we here introduce a partition of unity $\left\{j_{a}\right\}$, $2 \leqq \#(a) \leqq N$, over $X$, which will be employed in the proof of the proposition. The partition has the following properties.

(j.1) $j_{a} \in C^{\infty}(X), j_{a} \geqq 0$, is homogeneous of degree zero for $|x|>1$.

(j.2) $\quad \Sigma_{a} j_{a}(x)=1$ over $X$.

(j.3) $j_{a}(x) V_{\alpha}\left(r^{a}\right)=O\left(|x|^{-\rho}\right)$ as $|x| \longrightarrow \infty$ for $\alpha \not \subset a$ 。

We may further assume that $j_{a}$ takes the value one in a conical neighborhood of $S_{\alpha}$ for any two-cluster decomposition $a$ and that $j_{a}$ takes the value one or zero in a small conical neighborhood of $\hat{\omega}_{b}$ for all $b \in \Lambda^{c}$. Hence, $\nabla j_{a}$ vanishes in the regions as above. We can easily construct such a partition of unity.

Proof of Proposition 5.1. We prove the relation (5.1) for the + case only. We now write $\phi(t)=\exp (-i t H) f(H) \phi$ as

$$
\phi(t)=\sum_{a} \exp \left(-i t H_{a}\right) \exp \left(i t H_{a}\right) j_{a} \psi(t)
$$

with the partition $\left\{j_{a}\right\}$ above and take $g \in C_{0}^{\infty}(\boldsymbol{R})$ in such a way that $g=1$ on the support of $f$. As is seen from Theorem 3.1, $\phi(t)$ converges to zero weakly in $L^{2}(X)$ as $t \rightarrow \infty$ and also the operators $j_{a}\left(g(H)-g\left(H_{a}\right)\right)$ and $\left[g\left(H_{a}\right), j_{a}\right]$ are both compact on $L^{2}(X)$ by construction of the partition. Hence we have

$$
\phi(t)=\sum_{a} \exp \left(-i t H_{a}\right)\left\{g\left(H_{a}\right) \exp \left(i t H_{a}\right) j_{a} \psi(t)\right\}+o(1), \quad t \longrightarrow \infty .
$$

We represent the terms in brackets on the right side in the integral form. Then the proof is reduced to proving the strong convergence as $t \rightarrow \infty$ of the following two terms : 


$$
\begin{aligned}
& \varphi_{a 1}(t)=\sum_{c \notin a} \int_{0}^{t} g\left(H_{a}\right) \exp \left(i s H_{a}\right) j_{a} V_{a} \psi(s) d s, \\
& \varphi_{a 2}(t)=\int_{0}^{t} g\left(H_{a}\right) \exp \left(i s H_{a}\right)\left[\Delta / 2, j_{a}\right\rfloor \dot{\psi}(s) d s .
\end{aligned}
$$

By property (j.3), i $i$ follows from Proposition 3.2 that $\varphi_{a_{1}}(t)$ converges strongly in $L^{2}(X)$ as $t \rightarrow \infty$. To see the sirong convergence of $\varphi_{a 2}(t)$, we write the operator $\left[\Delta / 2, j_{a}\right]$ as

$$
\left[\Delta / 2, j_{a}\right]=q Q_{-1 / 4} Q_{1 / 4}\left[\Delta / 2, j_{a}\right]
$$

with $q \in C^{\infty}(X)$, where we should note that $q$ fulfilis all the assumptions in Proposition 4.1. Hence, by Corollary 3.4 and Proposition 4.1, we see that $\varphi_{a 2}(t)$ also converges strongly in $L^{2}(X)$ as $t \rightarrow \infty$. Thus the sysiem $H$ is shown to be asymptotically clustering and the proof is complete.

We now prove the main theorem.

Proof of Theorem 1.1. The theorem is proved by use of the standard inductive argument. The argument uses the asymptotic completeness for subsystem operators $H^{a}, a \notin \Gamma$, as inductive assumptions (the case $\#(a)=N-1$ has been already established) and the known fact ([12]) on the asymptotic completeness for $H^{a}, a \in \Gamma$, without electric fields. It should be noted that the pair interactions of $H^{a}, a \in \Gamma$, are all shori-range by assumption (VS). Let

$$
\mathscr{D}=\left\{f(H) \dot{\phi}: \phi \in L^{2}(X), f \in C_{0}^{\infty}(\mathbb{R})\right\} .
$$

The set $\mathscr{D}$ is dense in $L^{2}(X)$. Thus, to prove the theorem, it suffices to show that

$$
f(H) \phi \in \text { Range } W_{0}^{ \pm} \oplus \sum_{a \in \Gamma} \text { Range } W_{a}^{ \pm},
$$

because the ranges of wave operators are closed.

We again prove (5.2) for the + case only. We define the set $\Gamma_{a}$ as

$$
\Gamma_{a}=\{b: b \in \Gamma, b \subset a\}
$$

for $2 \leqq \#(a) \leqq N$. We note that $b$ belongs to $\Gamma$, if $b \subset a$ for some $a \in \Gamma$. Set again

$$
\psi(t)=\exp (-i t H) f(H) \phi, \quad f \in C_{0}^{\infty}(\mathbb{R}) .
$$

By inductive assumptions and the known fact above, it follows that for any $\varphi \in L^{2}(X)$,

$$
\exp \left(-i t H_{a}\right) \varphi=\exp \left(-i t H_{0}\right) \varphi_{0}+\sum_{b \in \Gamma_{a}} \exp \left(-i t H_{b}\right)\left(P^{b} \otimes I d\right) \varphi_{b}+o(1)
$$

as $t \rightarrow \infty$ for some $\varphi_{0}$ and $\varphi_{b}$ in $L^{2}(X)$. This, together with Proposition 5.1, yields that 


$$
\psi(t)=\exp \left(-i t H_{0}\right) \psi_{0}+\sum_{a \in \Gamma} \exp \left(-i t H_{a}\right)\left(P^{a} \otimes I d\right) \psi_{a}+o(1), \quad t \longrightarrow \infty,
$$

for some $\phi_{0}$ and $\psi_{a}$ in $L^{2}(X)$. Hence (5.2) is obtained from Proposition 2.1 at once and hence the proof is complete.

\section{References}

[1] Avron, J.E. and Herbst, I.W., Spectral and scattering theory of Schrödinger operators related to the Stark effect, Commun. Math. Phys., 52 (1977), 239-254.

[2] Graf, G. M., Asymptotic completeness for $N$-body short-range quantum systems: a new proof, Commun. Math. Phys., 132 (1990), 73-101.

[3] Herbst, I.W., Unitary equivalence of Stark effect Hamiltonians, Math. Z,, 155 (1977), 55-70.

[4] Jensen, A., Asymptotic completeness for a new class of Stark effect Hamiltonians, Commun. Math. Phys., 107 (1986), 21-28.

[5] Jensen, A. and Yajima, K., On the long range scattering for Stark Hamiltonians, J. reine angew. Math., 420 (1991), 179-193.

[6] Kato, T., Wave operators and similarity for some nonselfadjoint operators, Math. Ann., 162 (1966), 258-279.

[7] Kitada, H., Asymptotic completeness of $N$-body wave operators I. Short-range quantum systems, Rev. in Math. Phys., 3 (1991), 101-124.

[8] Korotyaev, E. L., On the scattering theory of several particles in an external electric field, Math. USSR Sb., 60 (1988), 177-196.

[9] Mourre, E., Absence of singular continuous spectrum for certain self-adjoint operators, Commun. Math. Phys., 78 (1981), 391-408.

[10] Perry, P., Scattering Theory by the Enss Method, Math. Rep., 1, Harwood Academic, 1983.

[11] Reed, M. and Simon, B., Methods of Modern Mathematical Physics III, Scattering Theory, Academic Press, 1978.

[12] Sigal, I. M. and Soffer, A., The $N$-particle scattering problem: asymptotic completeness for short-range systems, Ann. of Math., 125 (1987), 35-108.

[13] Tamura, H., Asymptotic completeness for $N$-body Schrödinger operators with short-range interactions, Commun. Partial Differ. Eqs., 16 (1991), 1129-1154.

[14] Tamura, H., Spectral and scattering theory for 3-particle Hamiltonian with Stark effect: asymptotic completeness, Osaka J. Math., 29 (1992), 135-159.

[15] Tamura, H., Spectral analysis for $N$-particle systems with Stark effect: nonexistence of bound states and principle of limiting absorption, Preprint, Ibaraki University, 1992.

[16] White, D., The Stark effect and long range scattering in two Hilbert spaces, Indiana Univ. Math. J., 39 (1990), 517-546.

[17] Yafaev, D., Radiation conditions and scattering theory for $N$-particle Hamiltonians, Preprint, Université de Nantes, 1992.

[18] Yajima, K., Spectral and scattering theory for Schrödinger operators with Starkeffect, J. Fac. Sci. Univ. Tokyo, Sec. IA, 26 (1979), 377-390. 